\title{
FORECASTING RECREATION DEMAND: AN APPLICATION OF THE TRAVEL COST MODEL
}

\author{
Richard A. Phillips and Jonathan I. Silberman*
}

\begin{abstract}
This paper presents a methodology for deriving forecasts of recreation (resort, etc.) demand based upon the standard travel cost model (TCM). To illustrate the technique, a sample TCM is derived to estimate visitation to the Virginia Beach, Virginia shoreline for the Summer, 1981 period. Using the estimated parameters from the model, visitation to Virginia Beach in 1990 is forecasted using data on income and population projections from the Bureau of Economic Analysis (U.S. Department of Commerce).

This paper develops a methodology for forecasting recreation demand using cross sectional data in the standard travel cost model (TCM). Several previous studies have illustrated the use of the TCM for estimating current demand for various recreational facilities. For example, Wetzstein and McNeely (1980) develop a TCM to estimate demand for winter recreation (skiing). Bowes and Loomis (1980), Ziemer et. al. (1980), and Strong (1980), estimate demand for trips to reservoir recreation areas. Allen (1981) estimates demand for camping recreation areas using the TCM. None of these studies, however, has demonstrated the usefulness of the TCM to regional planning authorities by presenting an effective method for deriving forecasts. For regions having an economic base of recreation demand, accurate forecasts are likely to be of considerable use to such authorities. In the first section, the basic TCM technique is presented. The second section applies the technique by developing a TCM for estimating visitation (recreation demand) to the Virginia Beach, Virginia
\end{abstract}

\footnotetext{
*Assistant Professor of Economics, Old Dominion University, and Professor of Economics, University of Baltimore. The data for this analysis were gathered in conjunction with a project funded by the U.S. Army Corps of Engineers, Norfolk, Virginia division. The authors wish to thank Garey G. Durden, Chairman, Department of Economics, Appalachian State University for his assistance on this project. Also to be acknowledged are two anonymous referees for their helpful comments.
}

shoreline. The third section presents the forecast methodology and results using the Virginia Beach TCM framework.

\section{The Travel Cost Model}

The forecast methodology is based upon the standard travel cost model. The TCM has been used extensively to value non-market goods such as public or quasi-public recreation areas. Barring the case of site entry fees, such facilities lack overt prices. Consequently, estimation of demand by traditional methods is not feasible. One method of resolving this problem is to develop a model which explains per capita visitation rates on the basis of travel costs from surrounding origin points. In this framework, travel cost is a proxy for price. It is assumed that individuals react to changes in travel costs in the same manner that they would respond to changes in overt prices such as entry fees. The result is a negative relationship between distance (and thus travel cost) and per capita visitation. Using this relationship, a demand function for the recreation services provided by the site may be derived.

In the basic version of the TCM, per capita visitation is a function of travel costs alone. In this paper we extend the basic TCM by adding additional explanatory variables. The model to be developed is given by the following expression:

$$
\frac{V_{i, j}}{N_{i}}=F\left(C_{i, j} ; Y_{i, j} ; S_{i, j}\right)
$$

$$
\text { where: } \begin{aligned}
\mathbf{V}_{\mathrm{i}, \mathrm{j}}= & \begin{array}{l}
\text { the number of individuals } \\
\text { from the ith origin zone } \\
\text { visiting the jth recreation } \\
\text { area; }
\end{array} \\
\mathrm{N}_{\mathrm{i}}= & \begin{array}{l}
\text { population of the ith origin } \\
\text { zone; }
\end{array} \\
\mathrm{C}_{\mathrm{i}, \mathrm{j}}= & \begin{array}{l}
\text { travel cost per party of } \\
\text { visitors from the ith origin } \\
\text { zone to the jth recreation } \\
\text { area; }
\end{array}
\end{aligned}
$$




$$
\begin{aligned}
\mathbf{Y}_{i, j}= & \text { median income level of } \\
& \text { visitors from the ith origin } \\
& \text { zone to the } j \text { th recreation } \\
& \text { area; and } \\
S_{i, j}= & \text { an index of the degree of } \\
& \text { substitutability of alter- } \\
& \text { native recreation sites to an } \\
& \text { individual from origin } i \\
& \text { visiting site } j .
\end{aligned}
$$

The explanatory variables used in the TCM are derived in a straightforward manner (detailed in Section II below). An exception is the travel cost variable. Economic theory suggests that travel cost be treated as a combination of direct (the variable cost of travel) and indirect (the opportunity cost of time) outlays. The direct costs of travel by private automobile are easily calculated. The indirect costs, however, will differ according to the individual's valuation of time. Resolution of this problem is, therefore, arbitrary to some extent. Most researchers have followed the suggestions of Cesario (1976) and McConnell and Strand (1981) in valuing travel time at some fraction of the average wage rate for the visitor's origin zone. This is based upon the presumption that recreation travel provides some satisfaction or utility apart from its objective: arrival at the site. Further, it is reasonable that time which is $a$ priori allocated to leisure should be valued at some fraction of the wage rate.

Given the availability of data, the relevant parameters for the above equation may be estimated. The predictive power of the model may be assessed by comparing the predicted and actual levels of total visitation (the sum of visitation from all origin zones) for the period. If properly specified, a high degree of accuracy is possible.

\section{Deriving a TCM for Virginia Beach, Virginia}

To illustrate the travel cost methodology, we present a sample TCM for overnight trips to the Virginia Beach, Virginia shoreline. Estimation of the model developed above requires sample data on: 1) the number of visits from a set of adjacent visitor origin zones; 2) an independent estimate of total current visitation; 3) the total population of each origin zone; 4) the travel cost from each origin zone, including round trip travel cost and the opportunity cost of travel time; and 5) median income of the visitors from each origin zone. The data sources and the generation of the required variables for the present example are detailed below:

\section{Per Capita Visitation}

Data on visitation per origin zone were obtained from the City of Virginia Beach. The city commissioned a survey in 1981 of all overnight visitors registering at nine hotels located in the prime area of the resort strip. Approximately 25,000 individuals from 450 different citycounty origin points were interviewed during the peak use period of the summer season: Memorial Day through Labor Day of 1981.

The independent estimate of total overnight visitation for 1981 (1.5 million visits) was obtained from the Virginia Beach Tourist Development Bureau. ${ }^{1}$ Given this estimate of total visitation, the origin sample was then used to generate the per capita visitation variable by origin. This was done by assuming that the percentage of the visitor sample from a given origin was equal to the percentage of total visitation from that origin. For example, if 5 percent of the visitor sample was from the City of Richmond then this implies that total overnight visitation from Richmond would equal 75,000 (.05 $\times 1.5$ million). To crente the per capita visitation variable, estimated visitation from each origin zone is divided by the population of that zone. Replication of this procedure for all zones completes the process. The procedure may be generalized by the following expression:

$$
\begin{aligned}
& \text { (2) Per-Capita = } \\
& \text { Visitation } \\
& \text { From Origin "i" } \\
& {\left[\begin{array}{l}
\text { Percentage of } \\
\text { Visitation } \\
\text { Sample From } \\
\text { Origin "i" }
\end{array}\right]\left[\begin{array}{l}
\text { Total Overnight } \\
\text { Visitation to } \\
\text { Virginia Beach }
\end{array}\right]} \\
& \text { Population of Origin " } i \text { " }
\end{aligned}
$$

\section{Travel Cost}

The travel cost variable equals the sum of the round trip cost of automobile operation and the opportunity cost of travel time. ${ }^{2}$ According to the American Automobile Association, the 
variable cost of operating an automobile in 1981 was $\$ .07$ a mile. This figure was multiplied by the round trip mileage from all origin points to Virginia Beach.

To calculate the opportunity cost of travel time, the duration of a trip in hours was computed by assuming a 50 mile per hour rate of speed (the AAA standard per hour). Valuation of travel time for each origin was set equal to $1 / 3$ of the average wage rate for adults and $1 / 2$ of the wage rate for each child. The wage rate for each origin was calculated by dividing median family income of that origin by the number of working hours in a year $(2,080)$. The number of adults and children were determined by assuming that the first two individuals in a party were adults whereas the remaining persons were children. The procedure may be generalized by the following expression:

(3) Travel Cost =

Per Party From

Origin " $i$ "

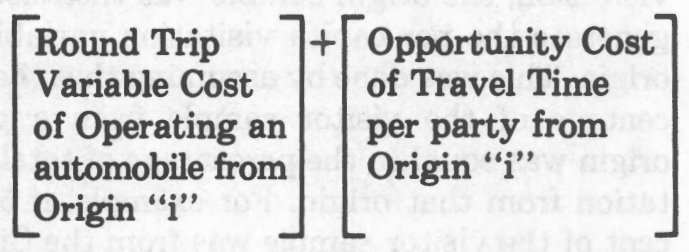

(4) Automobile =

Operating Cost

From Origin " $i$ "

$[.07]\left[\begin{array}{l}\text { Round Trip Mileage } \\ \text { From Origin "i" }\end{array}\right]$

(5) Opportunity Cost =

of Travel Time

from Origin " $i$ "

$\left[\begin{array}{l}\begin{array}{l}\text { Round Trip Mileage } \\ \text { from Origin "i" }\end{array} \\ 50\end{array}\right]\left[\begin{array}{l}\begin{array}{l}\text { Median Income } \\ \text { in Origin "i" }\end{array} \\ 2080\end{array}\right]$

$[.333]\left[\begin{array}{l}\text { Average Number } \\ \text { of Adults from } \\ \text { Origin "i" }\end{array}\right]+$

$\left[\frac{\begin{array}{l}\text { Round Trip } \\ \text { Mileage }\end{array}}{50}\right]\left[\frac{\text { Median Income }}{2080}\right]$

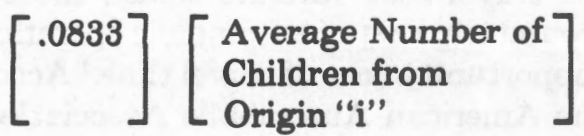

The travel cost coefficient is hypothesized to have a negative sign: as travel cost (price) increases via the effect of distance, per capita visitation will decline.

\section{Population and Income}

In the present example, population and median income data from each origin point were furnished by the Reuben H. Donnelley Advertising Service. This source of data is advantageous in that it is both complete and current. If such a data source is not available, however, the required information may be obtained from census data or other sources. The coefficient of the income variable is hypothesized to be positive: recreation service provided by the site is a normal good.

\section{Substitute Recreation Areas}

In the present example, the effect of substitute recreation areas on visitation to Virginia Beach is analyzed by means of dummy variables in the travel cost equation. Personal interviews with Virginia Beach tourism officials revealed that Ocean City, Maryland and Myrtle Beach, South Carolina are generally considered to be alternatives for Virginia Beach users. Therefore, dummy variables were created for each of these sites. The Ocean City dummy variable takes on the value of 1 if the ith origin is closer to Ocean City than Virginia Beach and 0 if otherwise; likewise, the Myrtle Beach dummy variable takes on a value of 1 if the ith origin is closer to Myrtle Beach than Virginia Beach and 0 otherwise. The coefficients of the two dummy variables are hypothesized to be negative: the closer either of these areas is to Virginia Beach for a given origin, the lower will be visitation to Virginia Beach from that origin. The summary statistics for the variables used in the Virginia Beach TCM are presented in Table 1.

Table 1

Summary Statistics for Variables Used in TCM

\begin{tabular}{lrc}
\hline \hline Variable & Mean & Standard Deviation \\
\hline Per-capita Visitation & .0544 & .0643 \\
Travel Cost & $\$ 122.22$ & $\$ 64.48$ \\
Median Income & $\$ 15,378$ & $\$ 3,609$ \\
Ocean City & .5038 & .5009 \\
Myrtle Beach & .0795 & .2711 \\
\hline \hline
\end{tabular}


The Virginia Beach TCM was estimated using the semi-log functional form. ${ }^{3}$ The sample size was truncated to exclude city-county origin points which were extreme outlyers. ${ }^{4}$ The final origin sample was 264 . The signs of all explanatory variables correspond to a priori expectations and are statistically significant at the 1 percent level of confidence. The value of the travel cost coefficient $(-.0050)$ suggests that visitation is relatively insensitive to price. Since travel cost represents the fixed component of the total cost of a visit (i.e., it is not affected by the duration of the visit), this result is to be expected. The adjusted $R^{2}$ value reveals that 42 percent of the variation in visitation rates is explained by the model. The results are detailed in Table 2.

Table 2

Travel Cost Model for

Virginia Beach Overnight Visitation, 1981 (Standard Errors in Parentheses Below Estimated Coefficient)

\begin{tabular}{lcc}
\hline \hline $\begin{array}{l}\text { Independent } \\
\text { Variable }\end{array}$ & $\begin{array}{c}\text { Dependent Variable: } \\
\text { Per Capita Visitation }\end{array}$ & $\begin{array}{c}\text { Log-Linear } \\
\text { Model }\end{array}$ \\
\hline Travel Cost & $-.005054^{*}$ \\
Median Income & $(.00117)$ \\
& $.000056^{*}$ \\
Ocean City & $(.00002)$ \\
& $-.797276^{*}$ \\
Myrtle Beach & $(.15544)$ \\
& $-1.78193^{*}$ \\
Constant & $(.23078)$ \\
$\mathbf{R}^{2}$ & -3.23116 \\
Sample Size & .423 \\
F.Value & 264 \\
\hline \hline
\end{tabular}

*Statistically significant at the one percent level or better

\section{The Forecast Methodology}

The estimated parameters from the TCM provide the basis for forecasting recreation demand. To obtain such forecasts, it is necessary to substitute estimates of future income and population levels for each origin into the model. Summing the calculated visitation from all origin points yields the desired forecast.

Since recreation demand is a normal good, it would be expected to increase at some positive rate as income increases. However, the rate of increase in visitation will not be uniform across origin points: some zones will experience higher than average rates of income growth whereas others will experience lower than average growth rates. Likewise the variability in population growth rates across origins will produce variability in per capita visitation growth rates across origins, holding constant the effects of income. Therefore, accurate forecasts of future recreation demand require independent estimates of future income and population from each origin. Aggregate data on these variables are not appropriate since inter-regional variation would not be identified. Unfortunately, local population and income forecasts are not consistently available. Some cities and counties derive these data while others do not.

An alternative source of forecast data is the U.S. Department of Commerce OBERS data series. The OBERS data provide estimates of population and real income growth rates for the years 1990,2000 , and $2030 .{ }^{6}$ In the present context, a difficulty with the OBERS data is that they are derived for SMSAs (and nonSMSA portions) as opposed to city-county origins. Therefore, the OBERS data do not coincide with the origin requirements of the Virginia Beach TCM.

This difficulty may be circumvented, however, by matching the coverage of OBERS data as nearly as possible to the original visitor survey origin points (not the truncated sample). In the present case, this operation resulted in a 90 percent matching of the citycounty origin points to SMSA or non-SMSA regions having OBERS projections. The approximation, while not perfect, is plausible given the cost of generating raw data. In this framework, the original visitor survey of $\mathbf{4 5 0}$ city-county origin points was transformed into 66 OBERS (SMSA and non-SMSA) origin points. ${ }^{6}$

The above operation yields a vector of 66 observations on travel cost, income, population, and Ocean City-Myrtle Beach dummy values. These data are then substituted into the original TCM to calculate visitation by OBERS origin point. To test the OBERS version of the TCM prior to its application for forecasting, simply compare predicted total visitation to actual visitation. In the present case, the model predicted total visitation (the sum of predicted visitation from all sample origin points) of $1,454,752$. Since actual visitation was approximately 1.5 million, the model performed quite well. The procedure is sum- 
marized by the following algorithms:

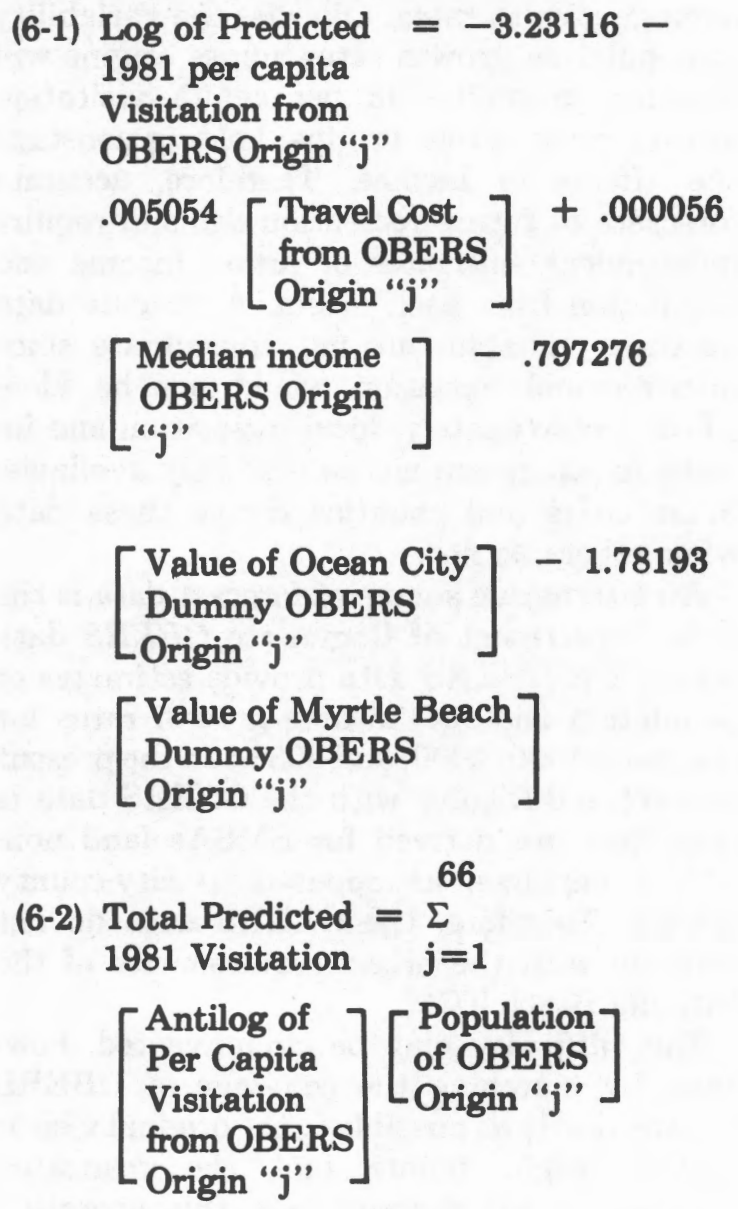

To forecast future visitation the OBERS projections for income and population are substituted into the model. ${ }^{7}$ Note that income growth influences visitation in both a positive and negative direction. The negative effect is due to the increase in the opportunity cost of travel time which rising income levels necessarily entail. Therefore, the income projection must also be substituted in the travel cost expression.

To derive the actual forecast, calculate the antilog of per capita visitation for each origin. This result is then multiplied by projected population for each origin. The sum of the result (visitation per origin) across all origins yields the desired forecast. The project is summarized by the following expression:

(7) Total

$\begin{array}{ll}\text { Visitation } & 66 \\ \text { Forecast }= & \sum \\ 1990 & \mathrm{i}=1\end{array}$

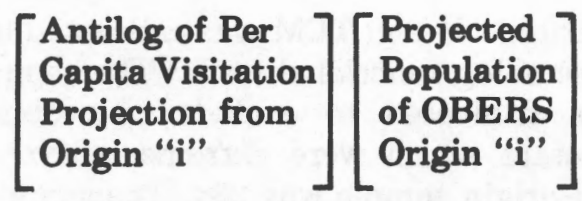

For the present example, the OBERS transformation generated a forecast for 1990 of $1,985,792$ overnight visits. This represents an increase of approximately 33 percent over the 1981 visitation level.

\section{Conclusion}

In this paper we have presented a method for deriving forecasts of recreation usage from the travel cost model. The forecast methodology utilizes existing OBERS data which are readily available. Thus, given the once only cost of developing a regional travel cost model, the cost of forecast generation is modest. To illustrate the technique, a sample TCM was generated for visits to the Virginia Beach shoreline during the summer of 1981 . The technique and associated data collection were explained in some detail. Using the parameter estimates generated from the TCM, the forecast method was then presented. A sample forecast of total overnight visitation to Virginia Beach for the year 1990 was then derived.

The strong points of this method are that: 1) it is not overly data intensive, and 2) the precision to cost ratio is likely to be high. Consequently, the method may be of considerable use to regional planning authorities. For regions in which recreation usage is an important component of the local economy, the method provides a framework for efficient long run decisions regarding resource allocation. In addition, relating future recreation demand to income growth by origin allows for promotional and marketing efforts to be targeted in a cost effective manner.

\section{FOOTNOTES}

\footnotetext{
'The estimate of total overnight visitation is based on: the total rooms available in the area, average length of stay, occupancy rates, and information from the Boardwalk Survey Research Report (1981). As demonstrated by the present example, an advantage of the TCM is that in many cases the required data are aiready available. The method is, therefore, relatively inexpensive.

'This version of the travel cost variable is appealing on theoretical grounds. However, for purposes of forecasting, if complete data on travel cost are unavailable, distance from the site is an acceptable proxy. Our experience sug-
} 
gests that the model performs nearly as well when distance (as opposed to travel cost) is used.

"The semi-log is the preferred functional form for estimating TCMs since it is a correction for homoskedasticity, a frequent problem in TCMs. For discussion of this point, see Ziener (1980) and Strong (1980).

-According to Smith and Kopp (1980), truncation of extreme outlyers is necessary on theoretical grounds. The further an origin point is from the recreation site, the greater is the probability that the trip was multi-purpose in nature. A necessary assumption for the TCM is that trips are single purpose (homogeneous); that is, they are specifically for recreation as opposed to permanent relocation, etc. As distance increases, so does the probability that trips are in fact nonhomogeneous. The exact point of truncation is, however, a question of judgment.

'The OBERS projections are prepared by the U.S. Department of Commerce Bureau of Economic Analysis. The projections cover economic activity in the U.S. by state, oconomic area, SMSA and state portions of these areas. Historical and projected values are presented for the period (1969-2030).

- For example, the Richmond, Virginia SMSA (OBERS origin point) is matched against the following city-county pairs from the original origin survey: Charles City, Richmond City, Chesterfield, Goochland, Hanover, Henrico, New Kent, and Powhatan Counties.

"Two clarifications on the use of OBERS projections must be noted. First, the OBERS data are forecasts of total population and total personal income by region. Since the TCM requires median income by origin, it is necessary to derive this from the given data. This is done by calculating the percentage change in the totals and applying it to the original median figures. The implicit assumption here is that income distribution has not changed. The second point relates to the three relative growth scenarios on which OBERS forecasts are derived: no change in share, low change in share, and moderate change in share. The first scenario assumes that the relative share of economic activity by state is constant over time whereas the others modify this assumption. For consistency, we used the no change in share figures throughout.

\section{REFERENCES}

Allen, Geoffrey, P., Stevens, Thomas H., and Barrett, Scott A. (1981). "The Effects of Variable Omission in the Travel Cost Technique," Land Economics, 57(May): 173-180.

Archer, B. (1977). Demand Forecasting in Tourism. Bangor, Wales: University of Wales Press.

"Boardwalk Survey Research Report," (1981). Unpublished report issued by the City of Virginia Beach Tourist Development Bureau.

Bowes, Michael D., and Loomis, John B. (1980). "A Note on the Use of Travel Cost Models with Unequal Zonal Populations," Land Economics, 56(Nov): 465-470.

Cesario, F. J. (1976). "Value of Time in Recreation Studies," Land Economics, 56(Nov): 32-41.

and Strand, Ivan (1981). "Measuring the Cost of Time in Recreation Demand Analysis: An Application to Sport Fishing," American Joumal of Agricultural Economics, 63(Feb): 153-156.

OBERS BEA Regional Projections (1980). Vols. 3, 4, and 5. U.S. Department of Commerce, Bureau of Economic Analysis.

Smith, V. Kerry, and Kopp, Raymond J. (1980). "The Spatial Limits of the Travel Cost Recreational Demand Model," Land Economics 56(Feb): 64-72.

Strong, Elizabeth J., "A Note on the Functional Form of Travel Cost Models with Zones of Unequal Populations," Land Economics 59(May): 247-254.

Wetzstein, Michael E., and McNeely, John G., Jr. (1980), "Specification Errors and Inference in Recreation Demand Models," American Journal of Agricultural Economics 62(Nov): 798-800.

Ziemer, R., Musser, W. N., and Hill, R. C. (1980). "Recreational Demand Equations: Functional Form and Consumer Surplus," American Journal of Agricultural Economics, 62(Feb): 136-141. 